This is an electronic reprint of the original article. This reprint may differ from the original in pagination and typographic detail.

\author{
Author(s): Karvonen, Anu; Kykyri, Virpi-Liisa; Kaartinen, Jukka; Penttonen, Markku; Seikkula, \\ Jaakko
}

Title: $\quad$ Sympathetic nervous system synchrony in couple therapy

Year: $\quad 2016$

Version:

Please cite the original version:

Karvonen, A., Kykyri, V.-L., Kaartinen, J., Penttonen, M., \& Seikkula, J. (2016).

Sympathetic nervous system synchrony in couple therapy. Journal of Marital and Family Therapy, 42(3), 383-395. https://doi.org/10.1111/jmft.12152

All material supplied via JYX is protected by copyright and other intellectual property rights, and duplication or sale of all or part of any of the repository collections is not permitted, except that material may be duplicated by you for your research use or educational purposes in electronic or print form. You must obtain permission for any other use. Electronic or print copies may not be offered, whether for sale or otherwise to anyone who is not an authorised user. 


\author{
FINAL DRAFT \\ Journal of Marital and Family Therapy \\ (2015, in press)
}

\title{
Sympathetic nervous system synchrony in couple therapy
}

\author{
Anu Karvonen \\ Virpi-Liisa Kykyri \\ Jukka Kaartinen \\ Markku Penttonen \\ Jaakko Seikkula \\ All authors are from University of Jyvaskyla
}

Anu Karvonen (M.A.), University of Jyvaskyla, Finland. Virpi-Liisa Kykyri (Ph.D.), University of Jyvaskyla, Finland. Jukka Kaartinen (Ph.D.), University of Jyvaskyla, Finland. Markku Penttonen (Ph.D.), University of Jyvaskyla, Finland. Jaakko Seikkula (Professor, Ph.D.), University of Jyvaskyla, Finland.

Conflicts of Interest and Source of Funding: There is no conflict of interest. All authors work in University of Jyvaskyla, Department of Psychology, Finland. The research was funded by the Academy of Finland, Human Mind Programme 2012-2016 (Decision No.: 265492).

Acknowledgements: We wish to thank Jarno Mikkonen for his contribution in coding MATLAB scripts, as well as Petri Kinnunen and Lauri Viljanto for technical help with autonomic nervous system measuring equipment.

Correspondence: Ms. Anu Karvonen, Department of Psychology, P.O. Box 35, FI-40014 UNIVERSITY OF JYVASKYLA; anu.karvonen@jyu.fi 


\section{SYMPATHETIC NERVOUS SYSTEM SYNCHRONY IN COUPLE THERAPY}

The aim of this study was to test whether there is statistically significant sympathetic nervous system (SNS) synchrony between participants in couple therapy. To our knowledge, this is the first study to measure psychophysiological synchrony during therapy in a multi-actor setting. The study focuses on electrodermal activity (EDA) in the second couple therapy session from 10 different cases (20 clients, 10 therapists working in pairs). The EDA concordance index was used as a measure of SNS synchrony between dyads, and synchrony was found in $85 \%$ of all the dyads. Surprisingly, co-therapists exhibited the highest levels of synchrony, whereas couples exhibited the lowest synchrony. The client-therapist synchrony was lower than that of the cotherapists, but higher than that of the couples. 


\section{INTRODUCTION}

Dialogue has been extensively studied in therapy settings, but interest has also started to emerge in nonverbal aspects of interaction, such as the role of facial expressions and body movements in therapy (Bänninger-Huber, 1992; Ramseyer \& Tschacher, 2011). In relation to this, researchers have acknowledged a relational rather than an individualistic view of the self, and emphasized the importance of "feeling felt" by others (Fishbane, 2001; 2007). Different terms have been used to describe this gut feeling of being connected; to name a few, Stern (1985) has referred to vitality affects, Damasio and Dolan (1999) background emotions, Siegel (2001) primary emotional processes, while Feldman (2012) has used the term bio-behavioral synchrony, and Porges (2007) has introduced the polyvagal theory to advocate the role of neural mechanisms in the regulation of biobehavioral processes. According to Lyons and Cromby (2010), there is a mutual interdependence of the body and the social world, but this connection has been recognized and studied only to a limited extent.

\section{The Relational Mind research project}

In the current research, we have adopted the term "embodied synchrony," arguing that attuning with others is a basic, often unconscious, process in which we resonate with our surroundings in our talk, body movement, facial expressions, and physiology - indeed with our whole embodied being (Seikkula, Karvonen, Kykyri, Kaartinen, \& Penttonen, 2015). Embodied synchrony does not refer only to similar reactions happening at exactly the same time; it can involve complementary synchrony and slow attuning (a lag in synchrony), and it can sometimes be guided or affected by cognitive effort.

Our current research project (The Relational Mind in Events of Change in Multiactor Therapeutic Dialogues) has been conducted at the University of Jyvaskyla in collaboration with 
four other universities in Europe. Our aim is to study attunement between several participants while trying to increase understanding of the embodied quality of therapeutic dialogues. Watching partners interact during stressful moments can tell us something about the couple's dynamics, aspects that may be missed in individual therapy (Solomon, 2009). It should be noted, however, that the therapists are informants on an equal footing with the clients, living their embodied experiences while being present to provide help as professionals.

In this paper the focus is on how clients' and therapists' sympathetic nervous system activity can synchronize during couple therapy sessions. By using psychophysiological recordings during therapy, it may be possible to detect bodily reactions which are not observable in behavior or talk, but which still reflect mental states (Hugdahl, 1995). In the project as a whole, several autonomic nervous system (ANS) variables have been recorded from both the clients and the co-therapists in the course of couple therapy. To our knowledge, this type of research has never previously been conducted with more than two participants, or during a real couple therapy session. Because of the large amount of data and the complexity of the setting, in this paper the focus is on electrodermal activity (EDA) synchrony at the beginning of therapy. Synchrony as a natural developmental process

Much of the research on non-verbal synchrony began through studying babies and their mothers in interaction. Human attachment develops through partners having both physiological and behavioral synchrony, and non-verbal synchrony helps to regulate the child's emotional states (Feldman, 2012; Siegel, 2001). The mirror neurons that have been found in the brain seem to be closely related to emotional resonance and a theory of mind, thus making intersubjectivity possible (Gallese \& Goldman, 1998; Rizzolatti \& Craighero, 2004; Iacoboni, 2009). The relational view of the human mind does not exclude intrapsychic issues (Beebe \& Lachmann, 
2003): thus as the child develops, mutual regulation becomes the skill of self-regulation. However, it is not just the relationship between the baby and the mother that is crucial, and multiple simultaneous relationships are apparent already in childhood (Hrdy, 2009; Reis, 2010). Very early on in our lives we start to move from a dyadic two-person interaction to interactions which include three or more persons (Fivaz-Depeursinge \& Philipp, 2014).

\section{Emotional contagion}

The ability of people to synchronize with each other is both a blessing and a curse (Fishbane, 2014). When one sees one's partner becoming angry, defensive, or accusatory, similar brain circuits may be activated in our brains - not just because of mirror neurons, but also because of emotional contagion. Hatfield, Cacioppo and Rapson (1994) describe this primitive emotional contagion as fairly automatic, unintentional, uncontrollable, and most often unconscious. Another person's emotions are not just sensed or understood, but partly caught by a process that is too complex and fast to be effectively mimicked (Doherty, 1997; Hatfield, Rapson, \& Le, 2011). Much in the way embodied synchrony was described above, emotional contagion can be manifested either as a similar response or as a complementary response. There has been interesting research suggesting that emotional contagion may be mediated by ancient evolutionary visual pathways, and that these can even bypass cortical vision (Tamietto et al., 2009). Facial expression and body expression stimuli evoke very similar reactions, suggesting that emotional contagion is not a consequence of motor mirroring, but rather caused by resonating to the affective meaning of stimuli. Nonetheless, emotional contagion is not deterministic - depending on the person's focus it can be fragile, or it can disappear completely (Englis, Vaughan, \& Lanzetta, 1982). 
Emotional contagion can be manifested as physiological synchrony when people are experiencing similar feelings. However, in the present study embodied synchrony is not seen as merely a primitive form of contagion. Embodied synchrony is a complex relational process which can also be regulated by cognitive effort. The start of couple therapy may be a situation in which clients are more focused on their own feelings rather than open to attuning to their spouse's feelings, and therapists have the education and experience to be aware of transference between clients and therapists.

\section{Embodied synchrony in couple therapy}

In everything that our brains engage in, resonance and synchrony provide "the underlying pulse," and interacting people are drawn into each other's emotional world (Hart, 2008). People are actually most likely to synchronize body movement with people they like, and this mimicking is not so much a cause of a positive relationship as a consequence of liking someone (Hatfield, Rapson, \& Le, 2011; McIntosh, 2006). New lovers can even buffer stress by increasing their parasympathetic activity when exposed to film clips which show negative relationships (Schneiderman et al., 2011).

In almost all effective couple therapies, affective processes seem to be crucial: attention has to be directed towards interrupting cycles of negative emotion, rebuilding emotional connections, and increasing positive affect (Harway, 2005; Gottman, 2002). Many couples fail to resonate with each other, and cannot be present with each other while they are in touch with their own core emotions (Solomon, 2009). Within therapy, misattuned early experiences can be manifested as clients being unable to read their own or others' emotions, and lacking the skill of self-soothing when they become upset (Fishbane, 2014). These clients want their partner to calm 
them down, and become disappointed when the partner's response to aggressive or difficult behavior is not what they wanted.

If therapists are skilled in noticing physiological and emotional reactions between spouses, and if they pay attention to their own reactions, they can obtain important information about the couple's relationship. Solomon (2009) describes noticing couples' pain and defenses in their bodies, and says he matches his bodily rhythm to that of the client, sometimes giving feedback concerning his observations. He goes on to explain that when powerful emotions come up and when the couple "cannot hear each other's pain," the therapist must attune empathetically, to downregulate the intensity of the situation. Clinicians often have experiences of their clients' emotions transferring to them; for example, their arousal level may decrease along with that of depressed clients (Hatfield, Rapson, \& Le, 2011).

Previous research on couples' synchrony

Studies concerning interpersonal psychophysiology have often been conducted via short non-therapeutic conversations. Levenson and Gottman $(1983 ; 1985)$ found that the physiological synchrony between couples was stronger when they were discussing problematic issues in relationships, as opposed to the normal events of the day; this synchrony was found to account for $60 \%$ of the variance in marital satisfaction. Furthermore, during the initial 1983 study they found that the more aroused the couple were (i.e. the higher their skin conductance), the greater was the decline in their marital satisfaction over the next three years. Unlike the present study, those recordings were not made during live couple therapy sessions; nor did they include therapists as participants. The couples' discussions were also not meant to include inherently happy or positive topics. 
Outside ANS research, studies focusing on hormonal synchrony have discovered that, for example, the cortisol levels of couples tend to synchronize, and that this hormonal synchrony is stronger for husbands who spend more time with their wives (Papp, Pendry, Simon, \& Adam, 2013; Liu, Rovine, Cousino Klein, \& Almeida, 2013). Feldman and colleagues (2012) found a correlation between plasma oxytocin level and newly formed couples' interactive synchrony, but they noted that other results were mixed. Much hormonal research has focused on the romantic beginning of a new relationship; by contrast, studies with couples who are facing problems have been rare.

\section{Sympathetic nervous system}

The autonomic nervous system is also called the involuntary nervous system, because it functions largely below the level of consciousness. It can further be divided into the sympathetic nervous system (SNS) and the parasympathetic nervous system (PNS). SNS activation has often been portrayed as the "fight/flight" response, with parasympathetic nervous system (PNS) activity being related to recovery, characterized as "rest/digest." During the child's development these sub-branches of the ANS become increasingly coupled (Schore, 1994). In body psychotherapy, SNS activation has been described in terms of feelings coming "up" (anger, fear, excitement, desire, hatred), while PNS activation is experienced as feelings that go "down" (shame, sadness, contentment) (Totton, 2005).

In the current study, SNS synchrony was measured using electrodermal activity (EDA) / skin conductance (SC). Skin conductance responses (SCR) are automatic in the sense that they are normally outside voluntary control (Hugdahl, 1995). Thus, for example, movement, rapid changes in respiration, or emotion-producing stimuli can cause changes in skin conductance 
(Greenfield \& Sternbach, 1972). Regarding therapy settings, it is interesting that most emotions tend to increase the level of skin conductance (Kreibig, 2010).

\section{Electrodermal activity concordance}

The analysis of EDA synchrony in this study is partly based on the methods developed by Marci and colleagues (2007). These researchers studied emotional distance and empathy in relation to EDA synchrony. In the study by Marci and Orr (2006), some patients had a normal interaction with an interviewer, whereas with others the interviewer made a conscious effort to minimize eye contact and nonverbal responses. Using a single index of EDA concordance throughout the interaction, Marci and Orr observed that the synchrony in the normal interaction was higher than in the emotionally distant condition. In addition, the patients' ratings of the interviewer's empathy were lower in the distant condition. These results indicate that a lack of eye-contact, coupled with nonverbal responses, can cause lower physiological synchrony, and that the inattentive person is likely to appear less empathetic to the partner in the interaction. Ramseyer and Tschacher (2011) obtained similar results by studying nonverbal synchrony between clients and therapists. They also found that the synchrony predicted relationship quality and therapeutic outcome.

Marci and colleagues (2007) used the same clients to study actual client-therapist dyads. According to ratings by external evaluators, the clients and therapists had significantly more positive social-emotional responses during high EDA concordance than during low EDA concordance. The reliability of the EDA concordance index was tested with hypothetical clienttherapist dyads, and the results were close to zero for random pairs. In contrast, the actual pairs had a clearly higher positive index. The therapy approach used in the study by Marci and colleagues was psychodynamic psychotherapy, and the clients in question had already had an 
average of 72 sessions with the therapist. In the current research project, SNS synchrony was studied both at the beginning of a therapeutic relationship and at the end of the process, and the therapy followed a dialogical/narrative approach.

Messina and colleagues (2013) also focused on the EDA concordance index as a measure of empathy, studying pseudo-clients and listeners (psychologists, therapists, or individuals without any kind of psychological education). EDA concordances were calculated for $0-4$ second lags, and the researchers noticed the initial concordance decreasing with psychologists but increasing with therapists as a function of time. Surprisingly, empathy as perceived by listeners did not correlate with the EDA concordances or with evaluations of empathy by clients. Messina and colleagues explained this result by referring to two forms of empathy - a sensory form based on somatic resonance, and a complex affective form connected to the social relationship. Their interpretation was that EDA concordance may involve more unconscious and non-verbal forms of empathy. Emotional empathy does seem to have distinct characteristics compared to cognitive empathy: it results in more mirroring of mental and bodily states, and engages the mirror neuron system better than cognitive empathy (Nummenmaa, Hirvonen, Parkkola, \& Hietanen, 2008).

It is quite possible that EDA synchrony in couple therapy represents a phenomenon with a wider scope than mere empathy. Again using EDA concordance indexes, Slovák and colleagues (2014) studied EDA synchrony between friends in a natural pub setting. They suggest that EDA synchrony reflects emotional reactivity between a pair, and that consistently high synchrony is related to the mutual engagement of the pair. This could explain some of the results of Levenson and Gottman (1983), according to which synchrony was higher during marital conflict discussions, as opposed to discussions on more neutral issues. In the Slovák et al. (2014) study, 
when one friend was secretly asked to ignore the partner in the interaction, synchrony actually increased if the naïve friend became irritated or tried to get answers from the passive friend. It has also been noticed that in comparison with either sad or happy stories, ambivalent stories increase the listener's EDA and heart rate, possibly because of the increased cognitive and interactional load (Voutilainen et al., 2014).

\section{Aims of the current research}

Previous research suggests that there can be physiological synchrony between two people who are interacting. Depending on the participants of the study, EDA synchrony has been connected to a number of variables, such as discussion of problematic issues, empathy, or emotional reactivity. It seems likely that EDA synchrony represents something related to overall engagement, involving a mutual increase or decrease in arousal and affect. If one looks at couples discussing stressful topics, the synchrony may correspond to unproductive "spiraling" (i.e. escalating recrimination or provocation). By contrast, if one looks at the synchrony between a client and a therapist, one may see empathetic attunement. Given the variety of manifestations, EDA synchrony per se is not considered to be "good" or "bad" in the current study.

Because EDA synchrony has not previously been analyzed during a real couple therapy session with therapists included also as participants, the first aim was to study whether there was statistically significant synchrony between dyads in a four-person setting. The second aim was to study differences in the level of synchrony between three subgroups, i.e. couple, co-therapist, and client-therapist dyads. It was hypothesized that synchrony would be found, and that the highest level of synchrony would be present between couples, due to their shared relationship history. The third aim was to investigate the time lag required for people to synchronize with each other. 


\section{METHODS}

\section{Participants}

The participants were ten Caucasian couples (20 clients) aged 27-61 years (mean 44) and ten therapists aged 31-64 years (mean 52). Two therapists worked together in the same room with each couple, following the normal procedure in the Psychotherapy Training and Research Center of the University of Jyvaskyla. The clients represented the ordinary clients met in the clinic, i.e. they were people who wished to participate in couple therapy because of marital crises or some other problems in their lives. In nine couples there was a female and a male client, and in one couple there were two female clients. The couples were asked to individually report reasons for seeking therapeutic aid. The reasons included mainly (i) problems in the couple's relationship (4 cases), (ii) problems outside the relationship (3 cases), (iii) violence in previous relationships (2 cases), and (iv) violence in the current relationship (3 cases).

Nine of the therapists were psychologists with several years of experience in clinical work, and eight of them were also licensed psychotherapists, as required by Finnish law (a minimum of three years of training). One therapist was not a psychologist, but was attending psychotherapy training. The therapists were assigned to cases on the basis of preliminary information on the couple and convenience in time scheduling, without consideration of the gender of the therapists. There were eight therapist dyads with a female and a male therapist, one dyad with two male therapists, and one dyad with two female therapists. Five out of the ten therapist dyads had

previously conducted therapy together, while for five dyads this study represented their first time as co-therapists. All the participants later got feedback through a "lifestyle assessment" (Firstbeat Technologies) involving three days of heart rate measuring.

\section{Procedure}


The research procedure was approved by the University of Jyvaskyla Ethical Committee. The couple therapy sessions lasted approximately 1.5 hours. The therapy approach was nonmanualized and the style could be described as narrative and dialogical. The therapists were instructed to work as they normally do, which often meant encouraging clients to speak of the salient issues in their lives at the moment. The therapists aimed to respond in ways which could elicit something new to contribute to the clients' stories. The sessions always contained reflective discussions between therapists, usually towards the end of each session.

In the first session, written informed consent was obtained from the participants. The sessions were recorded with six video cameras (a split-screen view covering the whole situation using two cameras, plus four cameras used to frame the face of each participant). The two therapists always sat next to each other, and the clients, too, sat next to each other; thus the formation resembled a circle around a table.

\section{Measurements}

During the second therapy session (in one case the third because of scheduling issues), psychophysiological recordings were conducted for the first time. These were followed by individual Stimulated Recall (SR) interviews (Kagan, Krathwohl, \& Miller, 1963) within one day of the session. In the SR interviews the participants watched four video extracts from the therapy session and the basic question of the interviewer was "What did you think or feel (also in your body) during that moment?" A similar measuring session followed by SR interviews was conducted towards the end of the therapy. The analysis in this article focuses on the electrodermal activity (EDA) in the first measuring sessions during therapy. However, it should be noted that the participants were wearing all the following devices: 
HEART rate monitoring. A day or two before the measuring session, the couple and the therapists attached heart rate monitors to their chest (Firstbeat Bodyguard, Firstbeat Technologies) and kept them on until the Stimulated Recall interviews were over. Two disposable electrodes were attached to the skin below the right collarbone and to the left side of the body below the heart, on the rib cage. The participants were instructed to remove the monitor only for shower/bath or swimming.

ELECTRODERMAL activity. Tonic skin conductance was recorded during the measuring sessions using two disposable electrodes $(\mathrm{Ag} / \mathrm{AgCl}$, Ambu Neurolone 710) placed on the palm of each participant's non-dominant hand, below the first and fourth digits. The electrodes were connected to a GSR module (Brain Products) determining the skin conductance with a DC instrumentation amplifier, using $0.5 \mathrm{~V}$ constant voltage. The signal was amplified in DC mode and low-pass filtered at $250 \mathrm{~Hz}$.

RESPIRATION. Fabric belts (BrainVision BP-BM-10 respiration belts), which stretch during respiration cycles, were fastened around each participant's lower chest.

An amplifier (BrainProducts Brainamp ExG 16) and a data acquisition program (BrainVision Recorder) were used to record EDA and respiration with a sampling rate of 1000 Hz. A marker unit was used to synchronize EDA and respiration to the video recordings. The participants were instructed to ignore the measuring devices and to act in their normal, natural way.

Questionnaires were used to obtain basic information on participants' medication, symptoms, previous psychiatric/physiological diagnoses, and treatments. At the start of every therapy session, the clients individually filled out Outcome Rating Scales (ORS) before the 
discussion started. At the end of every therapy session, both clients and therapists filled out Session Rating Scales (SRS) individually.

\section{STATISTICAL ANALYSIS}

\section{EDA concordance index}

To estimate the level of synchrony in participants' EDA, concordance indexes were calculated (see Marci et al., 2006, 2007; Messina et al., 2013). The data were resampled to $1 \mathrm{~Hz}$, and in order to detect change, the slope of the EDA was determined by calculating the sequential sample-to-sample differences $[(\mathrm{x}(\mathrm{t})-\mathrm{x}(\mathrm{t}-1)]$. The average slope of the EDA was determined using a moving 5-second window: The average slope value for the first 5 seconds of the EDA was calculated, after which the window was moved forward 1 second to calculate the following 5second slope value until all the data were analyzed. Pearson's correlations were calculated for the pairs using successive 15 -second windows at zero lag. A single session index was calculated from the ratio of the sum of the positive correlations during the entire therapy session, divided by the sum of the absolute value of the negative correlations during the session. A natural logarithmic transformation was calculated for this index, and by this means an EDA concordance index for the whole session was obtained. An index value greater than zero reflects predominately positive correlations during the session, whereas a value lower than zero indicates the dominance of negative correlations between the dyad. A value of zero represents an equal balance of positive and negative correlations between the dyad.

\section{EDA concordance indexes for different time lags}

The resulting EDA concordance index was also calculated for 15 different time lags, i.e. from -7 seconds to +7 seconds. Thus, regarding the concordance indexes between Client 1 and Client 2, if the highest value occurs during a positive lag, this means that Client 2's EDA follows 
Client 1's EDA. If the highest value occurs during a negative lag, this means that Client 1's EDA follows Client 2's EDA. If the highest value occurs during a zero time lag, the changes in EDA occur at the same time for both clients.

\section{Monte Carlo shuffling to assess statistical significance}

One problem concerning the concordance index is autocorrelation, which means that successive EDA values from a person are not independent of each other. To assess this, we determined a statistical significance level of $p<0.05$ to observe statistically significant concordances. In computing the Pearson correlations, time synchrony was detached between the participating dyad's signals: thus, for the first subject of the pair, successive 15-second time windows were used, but for the other subject a 15 -second time window was randomly selected from the recording session as a whole. Concordances were then computed as described above and sorted in ascending order. Finally, the 95\% point of the sequence was detected, and concordance was deemed to be statistically significant when the highest actual concordance was greater than the highest shuffled concordance of $\mathrm{p}<0.05$.

\section{Hypothetical pairs}

In addition to the above, we wanted to assess the problem of autocorrelation in a way that would not disrupt the "flow of therapy"; i.e. if there happened to be specific patterns of EDA because of the sessions themselves (such as high arousal at the start of therapy and decrease towards the end of therapy). Hence, EDA concordance indexes were calculated for hypothetical participant dyads. Ten new cases were formed from the data, with each participant drawn from a different couple therapy case.

\section{RESULTS}


Statistically significant EDA concordances were found in all couple therapy cases ( $\mathrm{p}<$ .05 ), but not necessarily between all dyads in the same case. Altogether, $85 \%$ of all dyads across cases showed a statistically significant EDA concordance index. In three cases all the dyads showed synchrony, and in the rest of the seven cases there was synchrony in four or five out of six possible dyads. In the cases where four out of six dyads had synchrony, the same person did not manifest significant synchrony with two different people (e.g. one client had synchrony with one therapist, but not with his/her spouse nor the other therapist).

\section{Case example}

An example from one case can be seen in Figure 1. Here, all the dyads except Lisa (all names changed) and Therapist 1 (T1) have a statistically significant concordance. The couple reported a wish for therapy because the spouses had different views on how to raise the children. Paul also reported a wish for help because the traumas in Lisa's previous abusive marriage were affecting their current relationship. During this session Lisa gave an account of traumatic experiences connected to bullying as a child at school, and subsequently during her years in working life, and by her ex-husband. She shed tears more than once; Paul remained silent while she was crying.

As shown in Figure 1, therapists T1 and T2 have a high EDA concordance index peaking at 0 second and +1 second time lags, while the client couple's index is slightly negative, rising to more positive at 0 seconds. This would indicate that the therapists shared a very similar arousal state throughout the session, i.e. becoming either relaxed or alert together, and reacting almost at the same time (with T2 "following" T1 rather than vice versa). The couple, on the other hand, had slightly more dissimilar than similar states of arousal: when one client relaxed, the spouse 


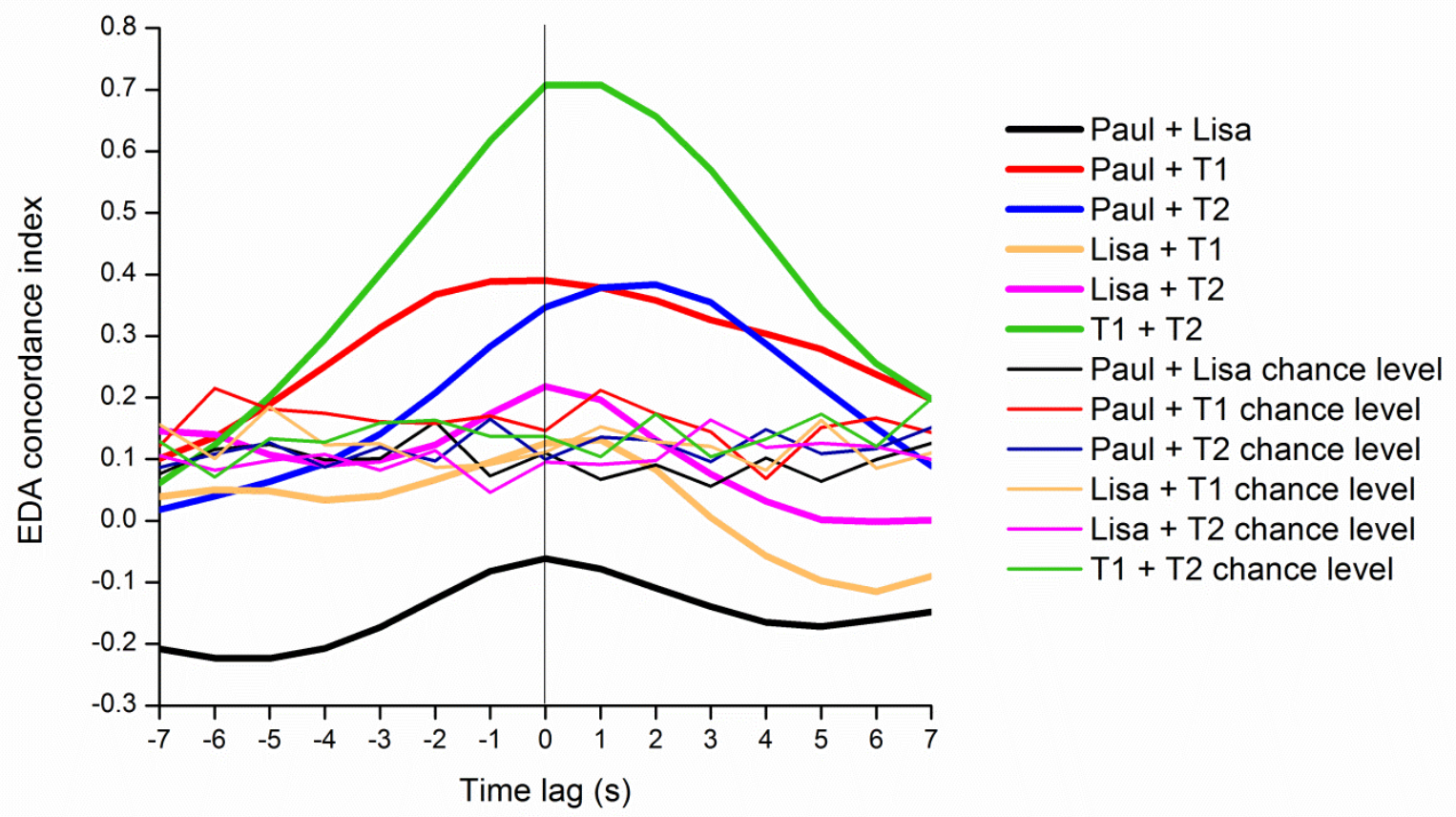

Figure 1. Example of EDA concordance indexes with 15 different lags in one couple therapy case. Each dyad has its own color, and the respective narrow colored lines represent the chance level values for each dyad $(p<0.05)$. 
became more alert (and vice versa). We assumed a one-tail statistical significance for chance level concordances, because to our knowledge, the kind of negative indexes seen with this couple have not previously been reported in dyadic studies. This was indeed the only dyad in our study with a negative highest concordance.

In her SR interview, Lisa re-lived situations of bullying when she was a child and cried throughout the interview: "I'm surprised how deep that came from. It has not previously caused this kind of reaction. How did this feel so hard now. I felt like a very very little girl. Hiding there behind the school. - - I feel it in my larynx, it squeezes. Kinda feels like hunching my shoulders. Even now I'm thinking about that school yard. - -." She said she could not understand why talking about these memories affected her so deeply. Paul, on the other hand, commented in his interview: “- - What should I say. I feel like Lisa has kinda stuck with the role of a victim. No matter what happens, she explains many things with similar issues. This was a familiar thing for me so I didn’t think any more about it - -.”

Lisa's synchrony with male Therapist 1 did not exceed chance-level synchrony, but there was slight statistically significant synchrony with female Therapist 2. In the SR Interviews T1 said he was keen to move on from the individual memories in order to deal with the relationship issues, while T2 felt the topic of bullying was an important topic for discussion when it came up, and she asked further questions about it.

\section{Group results (based on ten couples, ten therapists)}

The highest concordances from all the dyads were further divided into three normally distributed groups, namely couples (10), therapist dyads (10), and client-therapist dyads (40). The mean EDA concordance index for the couples was 0.22 , for the therapist dyads 0.65 , and for the client-therapist dyads 0.41. A one-way ANOVA showed that the levels of synchrony differed 
in these groups $(\mathrm{F}(2)=12,16, \mathrm{p}<.001)$; hence, post hoc tests were administered using the Bonferroni correction. The Eta-squared effect size was $\eta^{2}=0.27$, reflecting a large effect. A higher concordance was found between the therapist dyads than between the couple dyads (mean difference $0.43, \mathrm{p}<.001$, ES using Cohen's $d=2.36$, pooled SD) (Figure 2). The client-therapist dyads showed a lower concordance than the therapist dyads (mean difference $-0.24, \mathrm{p}<.01, d=$ 1.24), but a higher concordance than the couple dyads (mean difference $0.19, \mathrm{p}<.05, d=0.99$ ).

\section{Time lags}

In the 60 possible EDA concordances between dyads, the highest values between pairs occurred with time lags of -4 to +7 seconds. In $72 \%$ of the highest dyad concordances, the lag was between -1 and +1 seconds; hence the participants were synchronizing with each other very rapidly. Since negative and positive values depend on which of the two participants' EDA is moved backwards or forwards in time, it can be said that the participants' EDAs often reacted at the same time as or within a few seconds of lag. It was notable that the two therapists working together tended to show their best EDA synchrony at zero lag or at $+1 \mathrm{~s}$ lag (7/10 dyads). There was more variation in the mutual synchronization of the clients, with 5 out of 10 dyads showing the best synchrony at $0 \mathrm{~s}$ lag. $73 \%$ of the 40 different client-therapist dyads showed their best concordance at -1 to $+1 \mathrm{~s}$ lag.

\section{Hypothetical dyads vs. real dyads}

A one-way ANOVA was administered to test whether there were differences between the highest indexes of the actual cases, the hypothetical dyads (compiled from actual cases), and the Monte Carlo shuffled highest indexes. The actual dyads had, on average, a concordance index of 0.42 EDA; showing a statistically significant difference from both the average hypothetical dyad index of $0.22(d=1.05)$ and the average shuffled index of $0.18(d=1.35, \mathrm{p}<.001$ for both 


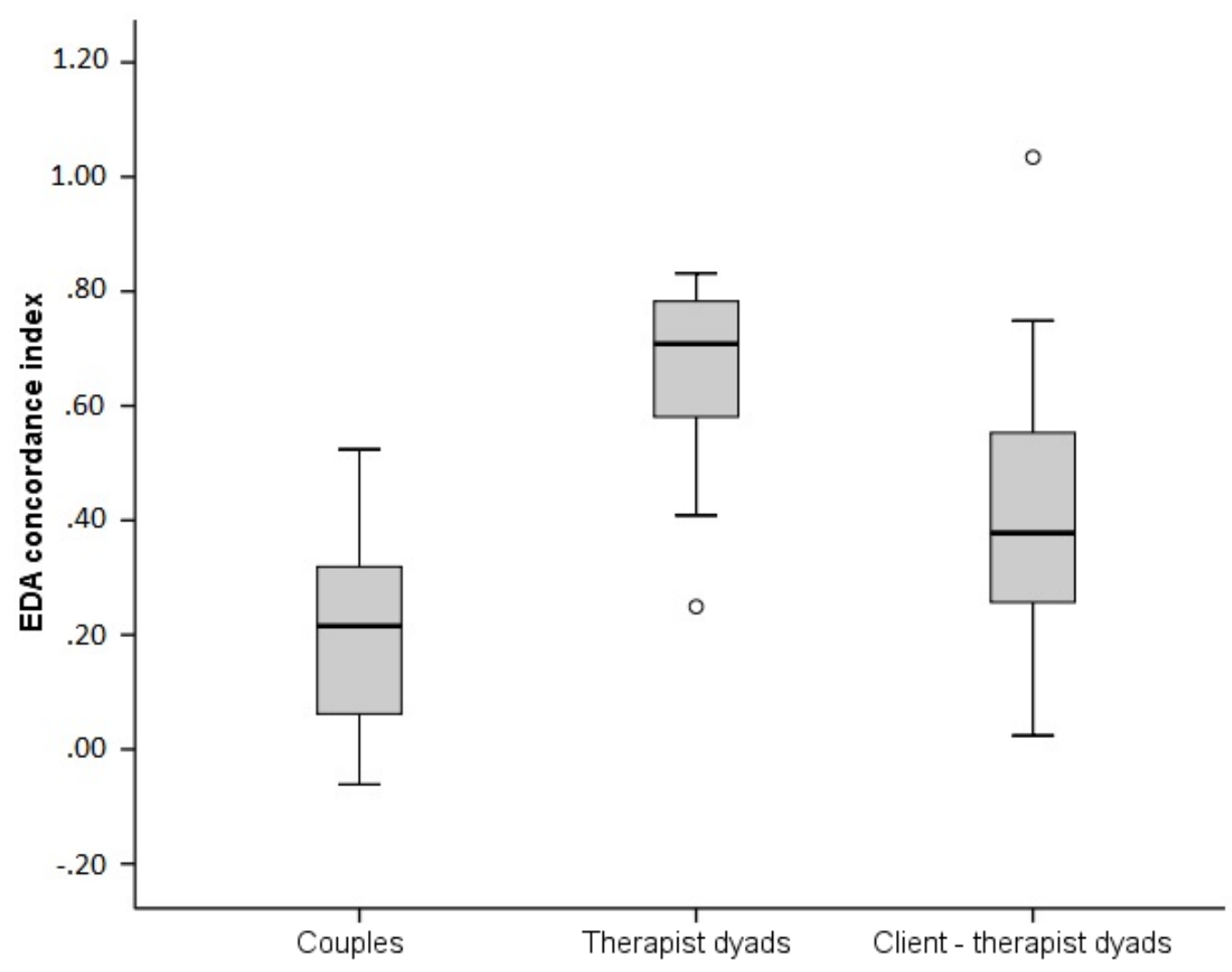

Figure 2. Boxplot figure of EDA concordance indexes between couples, therapists, and clienttherapist dyads. The boxes show the first and third quartile with the median in the middle. The whiskers represent the minimum and maximum, and the outliers are marked with a circle. 
conditions and $\eta^{2}=0.30$ ). The average hypothetical dyad index did not statistically differ from the average shuffled index $(\mathrm{p}=.50)$.

\section{Paper-and-pencil evaluations and EDA concordance}

Total ORS and SRS scores from the sessions for female clients, male clients, and cotherapists were correlated with the EDA concordance indexes using Pearson's correlation. No statistically significant correlation $(\mathrm{p}<0.05)$ was found between paper-and-pencil ratings and the level of EDA synchrony between dyads, even with bootstrapping (1000 samples). However, these observations should be considered as purely illustrative, since the sample was small and correlations had to be very strong to be considered significant. For example, the correlation between female and male clients' SRS scores was not statistically significant with a correlation of $.64(\mathrm{p}=.065)$.

\section{DISCUSSION}

The aim was to investigate whether sympathetic nervous system synchrony, as manifested by electrodermal activity (EDA) concordance between persons, would be present during couple therapy with co-therapists. Statistically significant synchrony was apparent in over four-fifths of the dyads. In comparing different groups it was observed that the co-therapists shared the highest level of EDA synchrony, whereas the client couples had the lowest synchrony. The level of synchrony between the client-therapist dyads was on average lower than that of the co-therapists, but higher than the synchrony between couples. There was no evidence of any universal "flow of therapy" effect on EDA synchrony, so in order to have high synchrony the people needed to be physically present with each other in the same room. We also discovered that the participants' EDA synchronized very rapidly, usually within a few seconds. 
Our results concerning client-therapist concordances were fairly similar to those of Marci and colleagues (2007). However, our enlarged multi-actor setting indicated that there were people who did not attune well with some of the others present in the situation. When one is talking with a single individual in a dyadic setting, one only has one addressee, so there is a need to maintain contact and to be understood. It may be that when several people are present, one can more freely choose the person to whom one directs one's words, and the person to look at. There is evidence that the affects portrayed by the face are crucial in regulating relationships (Benecke, Peham, \& Bänninger-Huber, 2005); hence, if one is not looking at someone, embodied information may be overlooked, with a consequent lack of bodily synchronization with that person.

In hypothesizing which participants would tend to have high EDA synchrony, we assumed that couples would show the highest attunement. In line with this hypothesis, it has been shown that e.g. cortisol synchrony is stronger when spouses spend more time together (Papp et al., 2013; Liu et al., 2013). Surprisingly our results did not support this assumption, since the highest levels of synchrony were observed between the two therapists. This probably happens because the co-therapists both focus their attention and effort on the clients rather than on each other. It has been shown that even brain area networks synchronize with people who are watching similar emotional events (Nummenmaa et al., 2012). Aspects such as similar training or one's role in the situation may also affect the level of synchrony. It was notable that the synchrony between the co-therapists was high, regardless of whether or not they had been working together previously.

The result that couples had the lowest synchrony is interesting, but hard to interpret without a comprehensive analysis of the interaction between the spouses. Quite possibly, the problematic relationship or the life situation had caused the couple to be more closed off from 
resonating with each other, in the manner described by Solomon (2009), i.e. not present while in a state of being in touch with their own core emotions. However, it is necessary to repeat that we do not regard low sympathetic nervous system synchrony between couples as good or bad in itself. With couples who are aggressively fighting and spiraling each other, synchrony is clearly not beneficial; however, if the couples are genuinely smiling and laughing together, that is positive synchrony. A lack of synchrony or a negative correlation in synchrony may sometimes be the best option when one person becomes very agitated and the other is trying to stay calm.

Averages do not represent individual variation, so one would need case studies to shed light on individual differences. Couples may have quite different problems that they wish to focus on; for example in our data there were some experiences of intimate partner violence, which may be associated with different physiological reactivity and synchrony patterns compared to other cases. However, there appeared to be no systematic differences in synchrony between couples that would correspond to the reasons for seeking therapy.

The case example of the bullied female client, Lisa, raised questions concerning the embodied qualities of traumatic experiences. According to Van der Kolk (1994), reminders of traumatic events are associated with chronic alterations in the stress response. The situation here, with Lisa reliving painful memories, and Paul thinking he had heard the story of "the victim" many times, might cause contrasting bodily states for them during the session: fight/flight vs. passive (or frustrated/bored) observer. By hunching shoulders, looking down, and avoiding eye contact, Lisa further limited the possibilities for others to synchronize with her embodied experience.

Because of autocorrelation, there was a need for some measure to ensure that synchrony was above chance level in the time series research. This was done by using Monte Carlo 
shuffling. In addition, we wanted to assess the "common flow of couple therapy," e.g. whether there would always at first be more arousal, with people relaxing towards the end. This was analyzed by compiling hypothetical cases, but there turned out to be no pattern that would explain SNS synchrony. In terms of actual values, in the study by Marci and colleagues (2007), the hypothetical dyads were somewhat closer to zero, probably because of a larger sample size. The average concordance for all the actual dyads in our study was similar to the concordances obtained by Marci and colleagues.

Even though the sample was small and the results must be regarded as illustrative, it was interesting that the paper-and-pencil evaluations of well-being, and evaluations of the therapy session, did not correlate with SNS synchrony. Messina and colleagues (2013) made the observation that listeners' evaluations of empathy did not correlate with the EDA concordance, while pseudo-clients and external raters seemed to be better evaluators of empathy. The answer may lie in SNS reactions being very automatic, unintentional, and often unconscious. They are at a different level from what is cognitively assessed, but they can be affected by, for example, the therapist's intentional focus on one client. For future research it would be interesting to code the alliance between dyads and compare that to the SNS synchrony, given that the therapeutic alliance has often been connected to the outcome of therapy (Martin, Garske, Davis, \& Katherine, 2000).

Our aim in the current study was to establish SNS synchrony as a real phenomenon in a couple therapy multi-actor setting. The co-therapists' high synchrony is fairly easily understood by the professional and situational requirements, but more research is needed to find out why couples may have low synchrony, and why a certain client may have more synchrony with a certain therapist. For this task it would be useful to study couple therapy cases individually, to 
look at shorter moments of interaction, and to combine different channels of information (physical movements of the body, facial expressions, dialogue, autonomic nervous system reactions, and SR interviews). Eventually it may even be possible to categorize certain interactions between couple therapy dyads according to their SNS synchrony, and to study the situations in which high synchrony seems to be beneficial or detrimental. The plan would also be to study whether synchrony is different at the end of therapy, and how the paper-and-pencil evaluations made during the therapy process relate to this information.

Our overall message for clinicians would be to attempt to focus on the fleeting, automatic bodily reactions in individual clients and between spouses, and to try to be aware of their own bodily states. By trusting in one's intuitive gut feelings, one can open up a path to becoming aware of unconscious reactions between partners, and it might be helpful, in a tentative way, to share some of these observations (Solomon, 2009). If therapists discover what nonverbal and verbal elements are connected to SNS synchrony, and how SNS synchrony is related to the alliance and the outcome of the therapy, they may be able to use these elements to enhance the therapeutic process. 


\section{REFERENCES}

Bänninger-Huber, E. (1992). Prototypical affective microsequences in psychotherapeutic interaction. Psychotherapy Research, 2(4), 291-306.

Beebe, B., \& Lachmann, F. (2003). The relational turn in psychoanalysis: A dyadic systems view from infant research. Contemporary Psychoanalysis, 39(3), 379-409.

Benecke, C., Peham, D., \& Bänninger-Huber, E. (2005). Nonverbal relationship regulation in psychotherapy. Psychotherapy Research, 15(1-2), 81-90.

Cacioppo, J. T., Bush, L. K., \& Tassinary, L. G. (1992). Microexpressive facial actions as a function of affective stimuli: Replication and extension. Personality and Social Psychology Bulletin, 18(5), 515-526.

Damasio, A., \& Dolan, R. J. (1999). The feeling of what happens. Nature, 401(6756), 847-847.

Doherty, R. W. (1997). The emotional contagion scale: A measure of individual differences. Journal of nonverbal Behavior, 21(2), 131-154.

Englis, B. G., Vaughan, K. B., \& Lanzetta, J. T. (1982). Conditioning of counter-empathetic emotional responses. Journal of Experimental Social Psychology, 18(4), 375-391.

Feldman, R. (2012). Bio-behavioral synchrony: A model for integrating biological and microsocial behavioral processes in the study of parenting. Parenting, 12(2-3), 154-164.

Fishbane, M. D. (2001). Relational Narratives of the Self. Family Process,40(3), 273-291.

Fishbane, M. D. (2007). Wired to connect: Neuroscience, relationships, and therapy. Family Process, 46(3), 395-412.

Fishbane, M. D. (2014). "News from Neuroscience”: Applications to Couple Therapy. In Critical Topics in Family Therapy (pp. 83-92). Springer International Publishing. 
Fivaz-Depeursinge, E., \& Philipp, D. A. (2014). The baby and the couple: Understanding and treating young families. Routledge.

Gallese, V., \& Goldman, A. (1998). Mirror neurons and the simulation theory of mindreading. Trends in cognitive sciences, 2(12), 493-501.

Gottman, J. M. (2002). The mathematics of marriage: Dynamic nonlinear models. MIT Press.

Greenfield, N. S., \& Sternbach, R. A. (1972). Handbook of psychophysiology.

Hart, S. (2008). Brain, attachment, personality: An introduction to neuroaffective development. Karnac books.

Harway, M. (2005). Handbook of couples therapy. John Wiley \& Sons.

Hatfield, E., Cacioppo, J. T., \& Rapson, R. L. (1994). Emotional contagion. Cambridge university press.

Hatfield, E., Rapson, R. L., \& Le, Y. C. L. (2011). 2 Emotional Contagion and Empathy. The social neuroscience of empathy, 19.

Hrdy, S. B. (2009). Mothers and others: the evolutionary origins of mutual understanding. Harvard University Press.

Hugdahl, K. (1995). Psychophysiology: The mind-body perspective. Harvard University Press. Iacoboni, M. (2009). Imitation, empathy, and mirror neurons. Annual review of psychology, 60, 653-670.

Kagan, N., Krathwohl, D. R., \& Miller, R. (1963). Stimulated recall in therapy using video tape: A case study. Journal of Counseling Psychology, 10(3), 237.

Kreibig, S. D. (2010). Autonomic nervous system activity in emotion: A review. Biological psychology, 84(3), 394-421. 
Levenson, R. W., \& Gottman, J. M. (1983). Marital interaction: physiological linkage and affective exchange. Journal of personality and social psychology, 45(3), 587.

Levenson, R. W., \& Gottman, J. M. (1985). Physiological and affective predictors of change in relationship satisfaction. Journal of personality and social psychology, 49(1), 85.

Liu, S., Rovine, M. J., Cousino Klein, L., \& Almeida, D. M. (2013). Synchrony of diurnal cortisol pattern in couples. Journal of Family Psychology, 27(4), 579.

Lyons, A. C., \& Cromby, J. (2010). Social psychology and the empirical body: Rethinking the relationship. Social and Personality Psychology Compass, 4(1), 1-13.

Marci, C. D., \& Orr, S. P. (2006). The effect of emotional distance on psychophysiologic concordance and perceived empathy between patient and interviewer. Applied psychophysiology and biofeedback, 31(2), 115-128.

Marci, C. D., Ham, J., Moran, E., \& Orr, S. P. (2007). Physiologic correlates of perceived therapist empathy and social-emotional process during psychotherapy. The Journal of nervous and mental disease, 195(2), 103-111.

Martin, D. J., Garske, J. P., \& Davis, M. K. (2000). Relation of the therapeutic alliance with outcome and other variables: a meta-analytic review. Journal of consulting and clinical psychology, 68(3), 438.

McIntosh, D. N. (2006). Spontaneous facial mimicry, liking and emotional contagion. Polish Psychological Bulletin, 37(1), 31.

Messina, I., Palmieri, A., Sambin, M., Kleinbub, J. R., Voci, A., \& Calvo, V. (2013). Somatic underpinnings of perceived empathy: The importance of psychotherapy training. Psychotherapy Research, 23(2), 169-177. 
Nummenmaa, L., Hirvonen, J., Parkkola, R., \& Hietanen, J. K. (2008). Is emotional contagion special? An fMRI study on neural systems for affective and cognitive empathy. Neuroimage, 43(3), 571-580.

Nummenmaa, L., Glerean, E., Viinikainen, M., Jääskeläinen, I. P., Hari, R., \& Sams, M. (2012). Emotions promote social interaction by synchronizing brain activity across individuals. Proceedings of the National Academy of Sciences, 109(24), 9599-9604.

Papp, L. M., Pendry, P., Simon, C. D., \& Adam, E. K. (2013). Spouses' cortisol associations and moderators: Testing physiological synchrony and connectedness in everyday life. Family process, 52(2), 284-298.

Porges, S. W. (2007). The polyvagal perspective. Biological psychology, 74(2), 116-143.

Ramseyer, F., \& Tschacher, W. (2011). Nonverbal synchrony in psychotherapy: coordinated body movement reflects relationship quality and outcome. Journal of consulting and clinical psychology, 79(3), 284.

Reis, B. (2010). A Human Family: Commentary on Paper by Elisabeth Fivaz-Depeursinge, Chloé Lavanchy-Scaiola, and Nicolas Favez. Psychoanalytic Dialogues, 20(2), 151-157.

Rizzolatti, G., \& Craighero, L. (2004). The mirror-neuron system. Annu. Rev. Neurosci., 27, 169192.

Schneiderman, I., Zilberstein-Kra, Y., Leckman, J. F., \& Feldman, R. (2011). Love alters autonomic reactivity to emotions. Emotion, 11(6), 1314.

Schore, A. N. (1994). Affect regulation and the origin of the self: The neurobiology of emotional development. Psychology Press.

Seikkula, J., Karvonen, A., Kykyri, VL., Kaartinen, J., \& Penttonen, M. (2015). The Embodied Attunement of Therapists and a Couple within Dialogical Psychotherapy: An Introduction 
to the Relational Mind Research Project. Family Process, Advance online publication doi: 10.1111/famp.12152.

Siegel, D. J. (2001). Toward an interpersonal neurobiology of the developing mind: Attachment relationships, "mindsight," and neural integration. Infant mental health journal, 22(1-2), $67-94$.

Slovák P., Tennent P., Reeves S., Fitzpatrick G. (2014). Exploring skin conductance synchronisation in everyday interactions. NordiCHI'14 (p. 511-520). ACM Press.

Solomon, M. (2009). Emotion in romantic partners: Intimacy found, intimacy lost, intimacy reclaimed. The healing power of emotion: Affective neuroscience, development \& clinical practice, 232-256.

Stern, D. N. (1985). The interpersonal world of the human infant.

Tamietto, M., Castelli, L., Vighetti, S., Perozzo, P., Geminiani, G., Weiskrantz, L., \& de Gelder, B. (2009). Unseen facial and bodily expressions trigger fast emotional reactions. Proceedings of the National Academy of Sciences, 106(42), 17661-17666.

Totton, N. (2005). New dimensions in body psychotherapy. McGraw-Hill Education (UK).

Van der Kolk, B. A. (1994). The body keeps the score: Memory and the evolving psychobiology of posttraumatic stress. Harvard review of psychiatry, 1(5), 253-265.

Voutilainen, L., Henttonen, P., Kahri, M., Kivioja, M., Ravaja, N., Sams, M., \& Peräkylä, A. (2014). Affective stance, ambivalence, and psychophysiological responses during conversational storytelling. Journal of Pragmatics, 68, 1-24. 